\title{
Label-free imaging of fatty acid content within yeast samples
}

\author{
N. Garrett, J. Moger \\ School of Physics, Biomedical Physics Group, University of Exeter, Exeter, Devon, EX4 4QL, \\ United Kingdom
}

\begin{abstract}
Fungi have been found to be an underlying cause of $70 \%$ of all plant and animal extinctions caused by infectious diseases. Fungal infections are a growing problem affecting global health, food production and ecosystems. Lipid metabolism is a promising target for antifungal drugs and since effective treatment of fungal infections requires a better understanding of the effects of antifungal agents at the cellular level, new techniques are needed to investigate this problem.

Recent advances in nonlinear microscopy allow chemically-specific contrast to be obtained non-invasively from intrinsic chemical bonds within live samples using advanced spectroscopy techniques probing Raman-active resonances. We present preliminary data using Stimulated Raman Scattering (SRS) microscopy as a means to visualise lipid droplets within individual living fungi by probing Raman resonances of the $\mathrm{CH}$ stretching region between $2825 \mathrm{~cm}^{-1}$ and $3030 \mathrm{~cm}^{-1}$.
\end{abstract}

Keywords: Raman, microscopy, lipid metabolism, fatty acid, yeast

\section{INTRODUCTION}

Although fungi play critical roles in virtually all terrestrial ecosystems, they are somewhat inconspicuous, often hidden within soil. They perform many nutrient-cycling and exchange functions, in addition to contributing greatly to the process of decomposition. It is often only when fungi are unwanted that their presence becomes known, such as candida overgrowth in oral thrush. Some species of fungi act as pathogens to crops and animals and are the underlying cause of many devastating diseases, leading to loss of crops, profits and contributing to famine. If the fungal diseases affecting the world's top five crops could be prevented from spreading, an estimated 600 million more people could be fed each year ${ }^{[1]}$. Given the increasing demands population growth is putting on crop production, it is clear that more research into effective fungicides is needed in the near future.

The growing resistance of some fungi to traditional fungicides has led to an increased demand for new, more effective anti-fungal treatments for both crops and animals, including humans ${ }^{[2]}$. Effective development of new fungicides requires a deep understanding of the sub-cellular interactions between the fungi and the drugs used to kill them. Lipid metabolism pathways provide a promising target for pharmaceutical drug development of fungicides, since interruption of lipid metabolism pathways can lead to rapid cell death ${ }^{[3]}$. One such potential pathway involves the enzyme Steroyl Coenzyme-A Desaturase (SCD), which is responsible for catalyzing a rate-limiting step in unsaturated fatty acid synthesis. SCD is responsible for the formation of a double bond in Stearoyl Coenzyme-A, enabling the production of oleic acid, a monounsaturated fatty acid, from stearic acid, a saturated fatty acid. Within the cytoplasm of fungi, synthesized fatty acids are stored in lipid droplets, acting as an energy store and providing the building blocks for membrane synthesis ${ }^{[4]}$.

Investigation of lipid metabolism of fungi has previously been undertaken with fluorescence microscopy using lipidspecific staining or green fluorescent protein (GFP) tagged proteins associated with lipid droplets ${ }^{[5]}$ and biochemical analyses of whole cell contents ${ }^{[4]}$. These methods enable detailed specifications on carbon chain length, degree of saturation, etc. to be readily obtained. However, there are drawbacks to invasive and indirect approaches that rely on staining, including potential side effects such as altered chemical and physical properties of the lipids, photo-induced

\footnotetext{
Imaging, Manipulation, and Analysis of Biomolecules, Cells, and Tissues XI, edited by

Daniel L. Farkas, Dan V. Nicolau, Robert C. Leif, Proc. of SPIE Vol. 8587, 858700

(C) 2013 SPIE · CCC code: 1605-7422/13/\$18 - doi: 10.1117/12.2002970
}

Proc. of SPIE Vol. $8587858700-1$ 
damages and uncertainties in the labeling/expression efficacy. Additionally, whole-cell biochemical analysis provides average data of all lipids present in the cell without any spatial or single-cell information.

Detailed, chemically-specific microscopic analyses of samples may be performed using spectroscopy-based methods, such as spontaneous Raman scattering microscopy. When a sample is illuminated with monochromatic light, a small proportion is inelastically scattered (Raman-scattered) relative to the incident light after interacting with the sample's molecular vibrations. When the frequency shift of the Raman-scattered light relative to the incident light is plotted as a function of intensity, a Raman spectrum is obtained, with peaks correlating to specific molecular vibrational modes. Although this technique provides a means to generate label-free chemical contrast with diffraction-limited spatial resolution, the major drawback to Raman spectroscopy is the small scattering cross section. Due to Raman scattering's low sensitivity, long acquisition times and relatively high laser powers are required for good signal-to-noise, therefore this technique is unsuitable for many investigations of sensitive, dynamic biological processes.

It is possible to generate much more intense Raman-based contrast from biological samples via the non-linear processes of Coherent Raman Scattering (CRS) microscopy ${ }^{[6]}$. In CRS microscopy, two incident lasers are used, a pump beam and a Stokes beam, which are made to overlap in time and space before being directed into a microscope, using lenses to focus the beams onto a sample. With this configuration, the non-linear nature of CRS microscopy enables threedimensional optical sectioning of biological samples with diffraction-limited spatial resolution to be achieved. Moreover, since near infra-red wavelengths can be used to provide the pump and Stokes beams, the risk of photo-damage is lessened and the penetration depth into scattering samples is maximized. To generate CRS contrast, the frequency difference between the pump $\left(\omega_{\mathrm{p}}\right)$ and Stokes $\left(\omega_{\mathrm{S}}\right)$ beams is tuned to match a Raman-active mode of interest $\left(\omega_{R}\right)$ within the sample such that $\omega_{\mathrm{p}}-\omega_{\mathrm{S}}=\omega_{\mathrm{R}}$. When this resonant condition is met, the excitation fields coherently drive the Ramanactive molecular bonds, thus generating an intense, coherent, non-linear Raman signal. This makes it possible to generate strong CRS contrast at video-rate acquisition times.

The first biological applications of CRS microscopy utilized coherent anti-Stokes Raman scattering (CARS), generating high signal-to-noise molecular contrast of $\mathrm{C}-\mathrm{H}$ vibrations in live bacteria ${ }^{[7]}$, hydrodynamics within amoeba ${ }^{[8]}$ and $\mathrm{C}-\mathrm{O}$ vibrations of DNA in human epithelial cells ${ }^{[9]}$. During the process of CARS, a blue-shifted anti-Stokes photon (at the frequency $\omega_{\mathrm{aS}}=2 \omega_{\mathrm{p}}-\omega_{\mathrm{s}}$ ) is generated, which may be spectrally isolated from the pump and Stokes beams using optical filters. The intensity of the anti-Stokes signal generated in CARS is proportional to the square of the number of chemical oscillators being probed, thus strong signal is obtained from samples rich in the molecular bond of interest. Since lipids are ubiquitous in biological samples and are highly abundant in C-H bonds, the much CARS imaging has involved obtaining contrast from the $\mathrm{C}-\mathrm{H}$ stretch region of the Raman spectrum ${ }^{[10]-[13]}$. Although lipid droplets within live fungi samples have been investigated with CARS ${ }^{[14]}$, the dispersive spectral profile of CARS complicates detailed analysis of the spectral features obtained with this method. Each peak in a spontaneous Raman spectrum will exhibit a different profile to those within its corresponding CARS spectrum, since each CARS peak comprises a resonant component, a non-resonant background and a mixing term. The characteristic dispersive spectral line shapes of each Raman-active mode in a CARS spectrum will result in congested, distorted spectra when the Raman peaks are close together, as in the fingerprint region.

An alternative CRS method has been recently reported which yields spectral profiles identical to those of spontaneous Raman scattering is stimulated Raman scattering (SRS) ${ }^{[15]}$. SRS probes the same molecular vibrations as those used in spontaneous Raman spectroscopy, but since it is a coherent process the signal detected is significantly stronger than that of spontaneous Raman scattering, which enables contrast to be obtained from even relatively weak Raman-active molecular vibrations. It is therefore possible to obtain detailed chemical information from single, live cells, using incident laser powers that are well within the tolerated range to avoid photo-thermal damage. The detection scheme of SRS differs from CARS, in that the detected signal does not come from a photon at a different wavelength to the incident pump and Stokes beams. When the frequency difference between the pump and the Stokes beam is tuned to match that of a Raman-active molecular vibration in the sample, stimulated Raman loss (SRL) whereby the intensity of the pump beam experiences a loss $\left(\Delta \mathrm{I}_{\mathrm{p}}\right)$ and stimulated Raman gain (SRG) whereby a the Stokes beam experiences a gain in intensity $\left(\Delta \mathrm{I}_{\mathrm{S}}\right)$, both occur simultaneously. SRL and SRG may be detected using modulation transfer detection, whereby the intensity of either the pump or the Stokes beam is modulated; the modulation is transferred to the other beam, and may be detected using a lock-in amplifier, with sensitivities of $\sim 1$ in $10^{6}$ photons. By choosing a modulation frequency above laser noise (>1 MHz) the signal can be detected with minimal noise. 
SRS microscopy is therefore a label-free technique because no extraneous dyes or fluorophores need to be added to the sample for contrast, providing chemically-specific and non-resonant background-free images. Additionally, the spectral profile obtained with SRS is identical to that of spontaneous Raman scattering, which makes comparison between the two techniques very straightforward. Because SRS is a coherent process, the Raman signal detected is much stronger than for spontaneous Raman scattering. The work outlined in this paper gives an overview of preliminary experiments performed using SRL microscopy to investigate the spectral profiles of lipid droplets within live yeast cells and determine the effects of a fatty acid desaturase inhibitor on these profiles.

\section{METHODS}

\subsection{Yeast cell sample preparation}

Cultures of yeast cells (Saccharomyces cerevisiae, S288C) were grown suspended in Vogel's minimal media and shaken at $80 \mathrm{rpm}$ at room temperature overnight. Fatty acid desaturase inhibitor dissolved in DMSO was diluted to a concentration known to be of $7.5 \mathrm{ppm}$ in Vogel's minimal media, with a final DMSO concentration of $20 \mu \mathrm{L}$ per mL of media. Cells were centrifuged and resuspended at a concentration of $\sim 1.0 \times 10^{6}$ cells per mL either with $7.5 \mathrm{ppm} \mathrm{SCD}-1$ inhibitor in minimal media (drugged group) or in minimal media alone (control group 1) or in minimal media with an equivalent concentration of DMSO as the drugged group (control group 2). After 1 hour incubation time at room temperature, $5 \mu \mathrm{L}$ of cell suspension was added drop-wise to glass coverslips coated in a thin layer of agarose gel. Each droplet was allowed to settle for 5 minutes before a second glass coverslip was placed on top, thus providing a means for keeping the cells stationary during microscopy.

\subsection{Saturated and unsaturated fatty acid mixtures}

Palmitic and palmitoleic acid mixtures at varying molar ratios were heated to $60{ }^{\circ} \mathrm{C}$ and homogenized before being sandwiched in between glass microscope coverslips, using a Parafilm spacer to ensure sample reproducibility.

\subsection{SRS microscopy}

The microscopy set-up used for SRS is illustrated in Fig. 1. A neodymium vanadate laser source (High-Q Picotrain, Hohenems, Austria) was used to provide a $1064 \mathrm{~nm}$ wavelength beam with a 6 ps pulse-width and a repetition rate of 76 $\mathrm{MHz}$, which was frequency-doubled to $532 \mathrm{~nm}$ to pump the Levante OPO (Levante Emerald, APE, Berlin). For SRS microscopy, the 'signal' beam output from the OPO was used as the pump beam, while a remnant of the $1064 \mathrm{~nm}$ beam from the Picotrain laser was used as the Stokes beam.

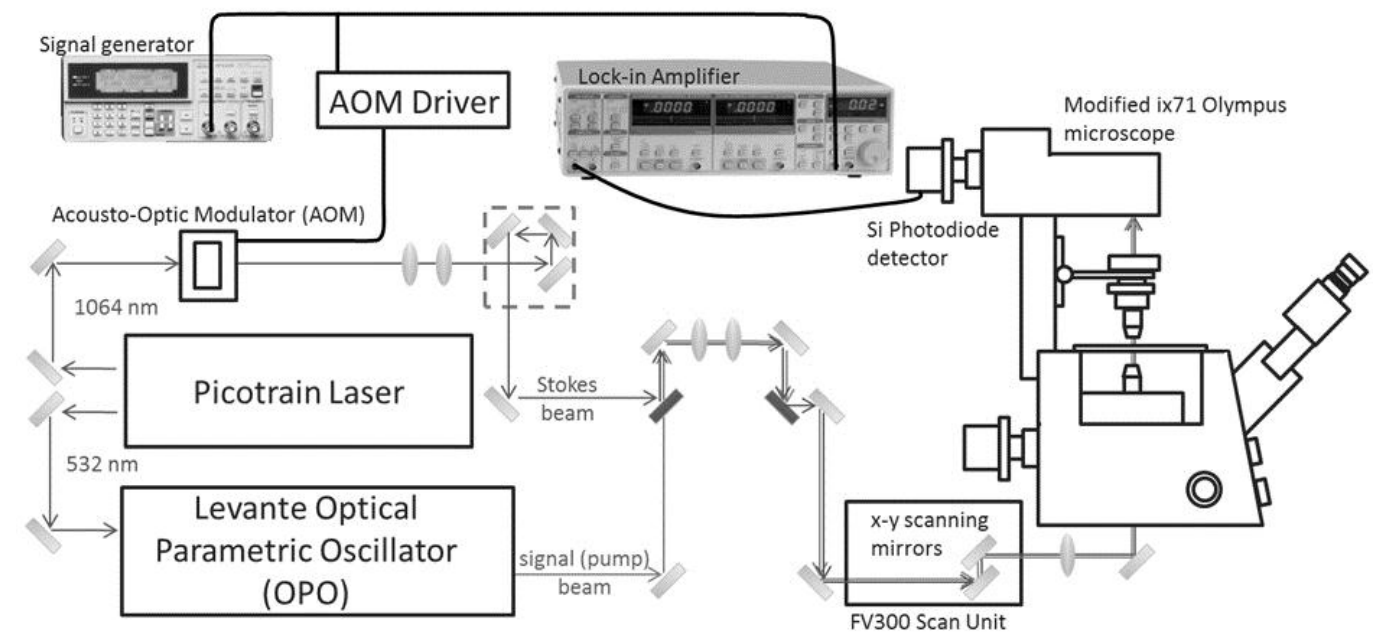

Figure 1. Schematic diagram of the optical microscopy set-up. 
The $1064 \mathrm{~nm}$ Stokes beam was amplitude-modulated by an acoustic optical modulator (AOMO 3080-197 Crystal Technology) driven by an 80 MHz driver (AODR 1080AF-A1F0-1.0, Crystal Technology). As illustrated in Fig. 2, when the pump and Stokes beams are focused onto the same spot on the sample and tuned such that the frequency difference between them matches a Raman-active molecular vibration in the sample, the amplitude modulation is transferred to the pump beam via the process of Stimulated Raman Loss (SRL). The SRL signal may then be detected by electronically demodulating the pump beam. A modulation frequency of $1.7 \mathrm{MHz}$ was chosen in order to prevent low-frequency laser noise from interfering with the SRS signal.

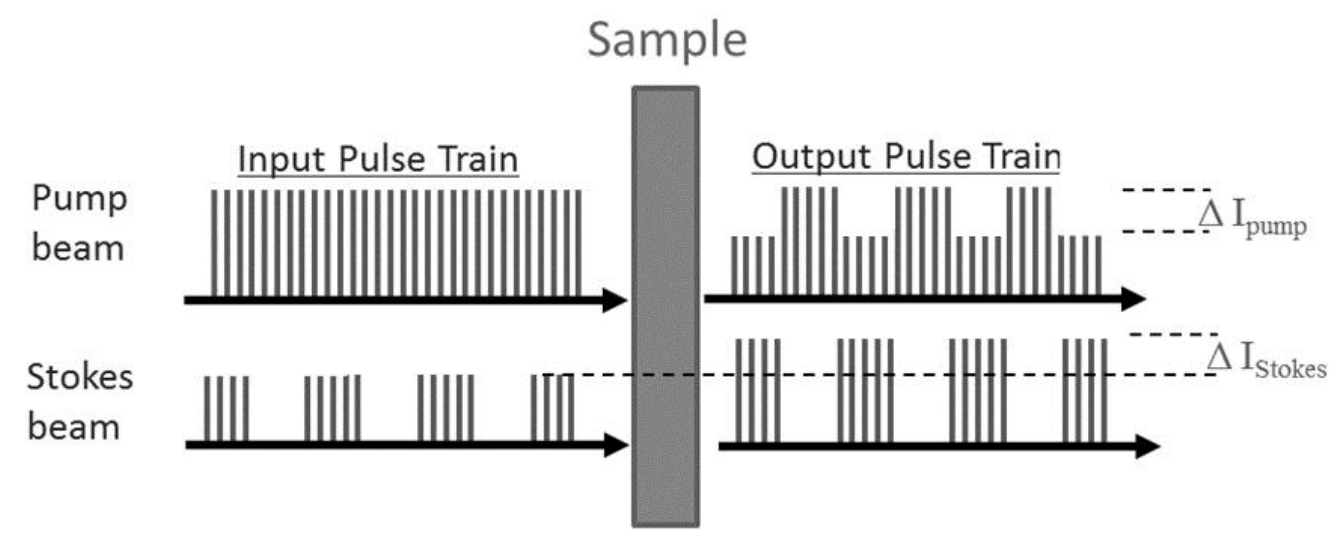

Figure 2. SRS laser beam intensity profiles when the energy difference between the pump and Stokes beams is tuned to a Raman-active molecular resonance.

The collinear Stokes and pump beams were directed into a modified commercial inverted microscope (IX71, Olympus, UK) and confocal scan unit (FV300, Olympus, UK). A water-immersion objective lens (UPLSAPO 60 x 1.2 NA, Olympus, UK) focused the Stokes and pump beams onto the sample, and a water-immersion condensing lens (Nikon Fluor $60 \mathrm{x}, 1.0 \mathrm{NA}$ ) collected and directed the transmitted light towards a band-pass filter to block the $1064 \mathrm{~nm}$ Stokes beam (CARS 890/220 nm, Chroma). The pump beam was then directed onto a wide-area silicon photodiode (FDS1010, Thorlabs, UK) with an applied reverse bias of $64 \mathrm{~V}$, which was positioned at the back-aperture of the condensing lens. The output from the Si photodiode is demodulated using a lock-in amplifier (SRS 844, Stanford Research Systems, UK) in order to detect the SRL signal at the modulation frequency of $1.7 \mathrm{MHz}$. The strong signal due to the laser pulsing at $76 \mathrm{MHz}$ was suppressed by filtering the output current with a low pass filter (mini-circuits, BLP-1.9+) and then terminating with a $50 \Omega$ resistor. The magnitude $(\mathrm{R})$ of the SRL signal output from the lock-in amplifier is fed into a computer and digitally analyzed.

\section{RESULTS AND DISCUSSION}

Mixtures of saturated and unsaturated fatty acids were investigated as a model fatty acid system using SRS, in order to provide a spectral basis for comparison to the spectra obtained in live yeast lipid droplets. Hyperspectral data were obtained by taking one image per data point in the spectrum, tuning the OPO to the required wavelength prior to the acquisition of each image. Fig. 3 (left panel) demonstrates the normalized SRL spectral profile of a mixture containing $80 \%$ palmitoleic acid and $20 \%$ palmitic acid (by molar mass), with peaks fitted to match the following Raman bands in the C-H stretch region: $2845 \mathrm{~cm}^{-1}, 2855 \mathrm{~cm}^{-1}, 2895 \mathrm{~cm}^{-1}, 2900 \mathrm{~cm}^{-1}$ and $2935 \mathrm{~cm}^{-1}$ which correspond with symmetric and antisymmetric $\mathrm{v}(\mathrm{C}-\mathrm{H})$ vibrations of $\mathrm{CH} 3$ and $\mathrm{CH} 2$ groups, while the $3006 \mathrm{~cm}^{-1}$ band is characteristic of unsaturated bonds in fatty acids resulting from $v(=\mathrm{C}-\mathrm{H})$ vibrations ${ }^{[16]-[18]}$.

Since the band at $3006 \mathrm{~cm}^{-1}$ has been successfully used as a marker of the degree of unsaturation of fatty acids in previous work ${ }^{[19]}$, we plotted the ratio of the SRL signal of $-\mathrm{C}(\mathrm{H} 2)$ at $2845 \mathrm{~cm}^{-1}$ to the SRL signal at $=\mathrm{C}(\mathrm{H} 2)$ at 3006 $\mathrm{cm}^{-1}$ for a range of mixtures of palmitic acid and palmitoleic acid. Because SRL signal is varies linearly with the concentration of the chemical bonds being investigated ${ }^{[20]}$, as expected the results (right hand panel in Fig. 3) yielded a linear relation between the ratio of SRL signal at $-\mathrm{C}(\mathrm{H} 2)$ to $=\mathrm{C}(\mathrm{H} 2)$ and the relative percentage of saturated fatty acids in the mixture. 

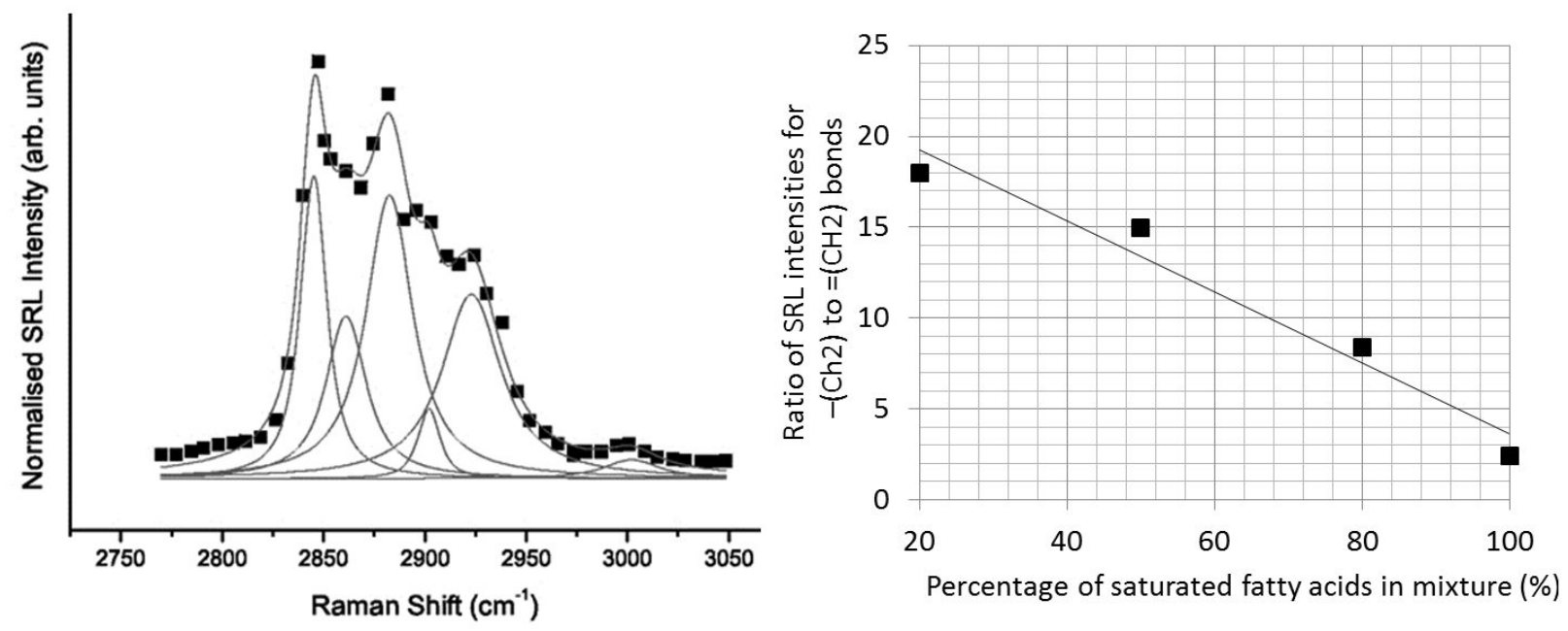

Figure 3. Left: Hyperspectral SRL data obtained from a mixture of $80 \%$ palmitoleic acid and $20 \%$ palmitic acid (by molar mass). Right: The correlation between the ratio of the SRL intensities for the peaks fitted at $2845 \mathrm{~cm}^{-1}$ to $3006 \mathrm{~cm}^{-1}$ and the percentage of saturated fatty acids in the mixture.

Live yeast cultures were exposed to a fatty acid desaturase inhibitor which is known to prevent the formation of unsaturated fatty acids within yeast, eventually leading to cell death. Control cell groups were also investigated in order to compare the effect of the inhibitor drug on the relative levels of saturated and unsaturated fatty acid signal in the SRL spectral profiles of lipid droplets within the live cells. The sub-micron spatial resolution of SRL enabled lipid droplets within the yeast cells to be pin-pointed (as illustrated in Fig. 4). Acquisition of SRL spectral profiles from these lipid droplets across the $\mathrm{C}-\mathrm{H}$ stretch region $\left(2775-3060 \mathrm{~cm}^{-1}\right)$ was complicated by the normal motion of the lipid droplets within the cells' cytoplasm. This issue was partly overcome by acquiring the images rapidly (acquisition times of $<10$ minutes per spectrum) and deflecting a portion of the light transmitted through the sample onto a separate photo-diode to provide a white-light transmitted image, which could be used to track the motion of cells and organelles throughout the course of the experiment. Lipid droplet spectral profiles were obtained for a minimum of 10 cells per group, and at least 3 lipid droplets per cell.
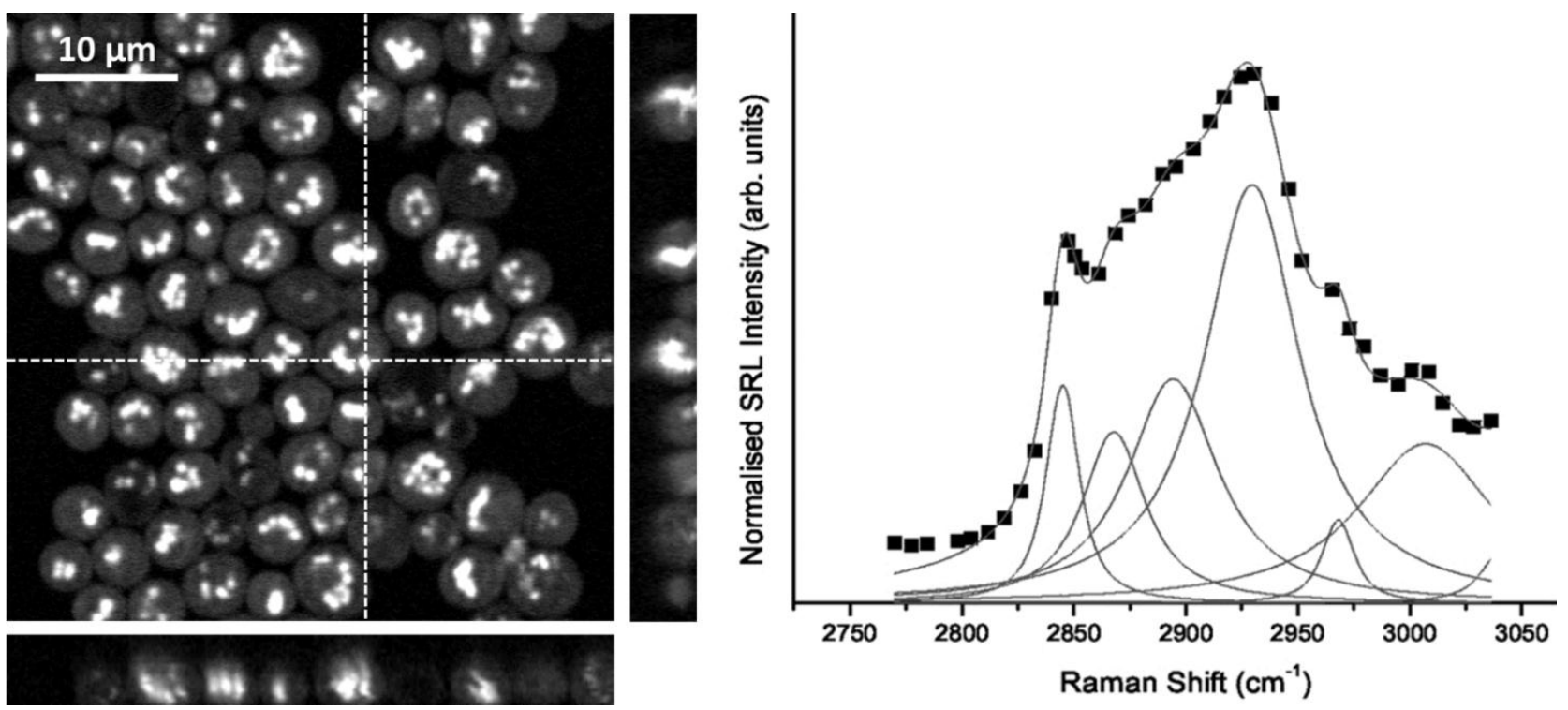

Figure 3. Left: Three-dimensional rendering of an SRL depth-stack taken of a control group of live yeast cells. Right: Hyperspectral SRL profile of the lipid droplets within the control cell group. 
Hyperspectral data for each of the three cell groups were plotted in the manner illustrated in the right panel of Fig. 4, and the same peaks in the $\mathrm{C}-\mathrm{H}$ stretch region of the Raman spectrum as used in our previous analysis of fatty acid mixtures were fitted to these data sets. We took the ratio of the peak heights for the peaks fitted at $2845 \mathrm{~cm}^{-1}$ to $3006 \mathrm{~cm}^{-1}$ and determined the differences in these ratios for control group 2 and the drugged group, as a percentage change relative to that for control group 1. Our data showed that for the control group exposed to DMSO-containing media, the percentage change in the ratio of unsaturated fatty acid signal to saturated fatty acids signal relative to the other control group was -2 $\%+/-3 \%$. This indicates that the addition of $20 \mu \mathrm{L}$ of DMSO per mL of media had no significant effect on the fatty acid content of the lipid droplets. For the drugged group, the percentage change in the ratio of unsaturated fatty acid signal to saturated fatty acid signal relative to the control group was $-55 \%+/-4 \%$. This result demonstrates that SRL can be used to monitor the change in proportions of unsaturated fatty acids relative to saturated fatty acids within lipid droplets. Being able to monitor these changes at sub-micron resolution in situ in live cells presents a significant advantage over traditional methods for monitoring cell lipid content, such as gas chromatography mass spectroscopy, which cannot separate lipid droplets from the cell membranes.

\section{CONCLUSIONS AND FUTURE WORK}

In principle, since SRS signal is linearly proportional to the concentration of the number of chemical bonds in the sample, it should be possible to use SRS to perform quantitative analysis of the degree of saturation within lipid droplets. However, the C-H stretching modes are sensitive to both temperature and phase transition ${ }^{[16]}$ which can complicate this analysis. Additionally, lipid droplets also contain small amounts of protein, which also contribute to the $\mathrm{C}-\mathrm{H}$ spectral profile ${ }^{[4]}$. However, the work outlined in this preliminary study demonstrates that it is possible to use SRL for comparative analysis between samples, in order to elucidate subtle chemical changes. Using this technique, it will be possible for in situ monitoring of fatty acid metabolism, allowing more detailed information to be gleaned about the efficacy of anti-fungal drugs. In future, work will be undertaken to further probe the interactions between anti-fungal drugs and live fungi, as both a function of incubation time and drug concentration.

\section{REFERENCES}

[1] M. C. Fisher, D. a Henk, C. J. Briggs, J. S. Brownstein, L. C. Madoff, S. L. McCraw, S. J. Gurr, Nature 2012 , $484,186$.

[2] J. J. Steffens, E. J. Pellt, M. Tien, n.d., 348.

[3] L. N. Nguyen, J. D. Nosanchuk, Communicative \& Integrative Biology 2011, 4, 245.

[4] K. Grillitsch, M. Connerth, H. Köfeler, T. N. Arrey, B. Rietschel, B. Wagner, M. Karas, G. Daum, Biochimica et biophysica acta 2011, 1811, 1165.

[5] K. Natter, P. Leitner, A. Faschinger, H. Wolinski, S. McCraith, S. Fields, S. D. Kohlwein, Molecular \& cellular proteomics : $M C P \mathbf{2 0 0 5}, 4,662$.

[6] W. Min, C. W. Freudiger, S. Lu, X. S. Xie, Annual Review of Physical Chemistry 2011, 62, 507.

[7] A. Zumbusch, G. R. Holtom, X. S. Xie, Physical Review Letters 1999, 82, 4142.

[8] E. Potma, W. P. de Boeij, P. J. van Haastert, D. a Wiersma, Proceedings of the National Academy of Sciences of the United States of America 2001, 98, 1577.

[9] A. Volkmer, J. X. Cheng, X. S. Xie, Phys. Rev. Lett. 2001, 8702, 023901.

[10] C. Heinrich, A. Hofer, A. Ritsch, C. Ciardi, S. Bernet, M. Ritsch-Marte, Optics Express 2008, 16, 2699.

[11] J. X. Chen, A. Volkmer, L. D. Book, X. S. Xie, Journal of Physical Chemistry B 2002, 106, 8493.

[12] X. L. Nan, J. X. Cheng, X. S. Xie, Journal of Lipid Research 2003, 44, 2202.

[13] T. T. Le, S. Yue, J.-X. Cheng, Journal of lipid research 2010, 51, 3091.

[14] C. Brackmann, J. Norbeck, M. Åkeson, D. Bosch, C. Larsson, L. Gustafsson, A. Enejder, Journal of Raman Spectroscopy 2009, 40,748.

[15] C. W. Freudiger, W. Min, B. G. Saar, S. Lu, G. R. Holtom, C. W. He, J. C. Tsai, J. X. Kang, X. S. Xie, Science 2008, 322, 1857.

[16] M. C. Costa, M. Sardo, M. P. Rolemberg, P. Ribeiro-Claro, A. J. a Meirelles, J. a P. Coutinho, M. a Krähenbühl, Chemistry and physics of lipids 2009, 157, 40.

[17] V. Baeten, P. Hourant, Journal of agricultural ... 1998, 8561, 2638.

[18] M. Maier, D. de Faria, M. Boschín, S. Parera, Arkivoc 2005, 2005, 311.

[19] E. Li-Chan, Trends in Food Science \& Technology 1994, 5, 3.

[20] P. Nandakumar, a Kovalev, a Volkmer, New Journal of Physics 2009, 11, 033026. 